\title{
PERFORMANCE OF ENERGY BALANCED TERRITORIAL PREDATOR SCENT MARKING ALGORITHM BASED ON LEACH PROTOCOL
}

\author{
Radha B Khandre ${ }^{1}$, Ramesh Patil ${ }^{2}$ \\ ${ }^{I} P G$ Student, ECE Department, Guru Nanak Dev Engineering College Bidar, Karnataka, India \\ ${ }^{2}$ Associate Professor, ECE Department, Guru Nanak Dev Engineering College Bidar, Karnataka, India
}

\begin{abstract}
Sensor node is a node in wireless sensor network (WSN) that is capable of sensing information, processing and communicating with each other node in the network. As the energy available in the sensor nodes is limited. So while designing WSN, the main concentration has to be taken part in network life time by using the energy efficient sensor node placement in many network applications. So energy efficient operation is very important, as the sensor nodes are depends on battery for operation to be carry out. It is difficult or replaces charge battery, for this sensor nodes function and operation in WSN should become energy efficient. To overcome this problem new biologically inspired technique has been introduce the algorithm and called as Energy balancing Territorial Predator Scent Marking Algorithm using LEACH protocol in WSN.TPSMA is used for sensor node schemes in terms of coverage ratio, network life time by this network efficient network can be achieved. This is based on the scent marking behavior, predator will scent mark the area due to certain factors such as resources. Sensor node will identify it's monitored location based on their marked territories that imitate the scent matching behavior in TPSMA. The performance of the proposed technique improved and compare with other Swarm Intelligence technique. At the end of this paper we highlighted the future work of this paper.
\end{abstract}

Keywords: Wireless sensor network, Swarm Intelligence, Low energy adaptive clustering hierarchy, and TPSMA etc...

\section{INTRODUCTION}

In wireless communication technology, the WSN become most evolving field of the development. WSN is a highly distributed, small size, light weight. Sensor function is capable of sensing, processing and data communication. Scent territorial marking also known as territorial marking. It is a behavior used by animals to identify their territory [8]. Animal scent in the form like urine, faeces mark the frequently to assist in communication of the information.

Network coverage area, network life time and connectivity in the wireless sensor network largely depend on deployment of sensor node. In order to make sensor node to be energy efficient for becoming network life time is maximum. WSN area becomes most popular due to increase in applications of networks. The autonomous nodes are distributed in territorial area for monitoring the environment such as pressure, temperature, sound, vibration, motion. Application of WSN are agriculture monitoring, target tracking, health care monitoring system. The Swarm intelligence is emergent collective, intelligence of groups of small agents. It is local interaction of many simple agents to achieve a global goal. Swarm Intelligence (SI) is the collective behavior of decentralized, self organized systems, natural or artificial. The concept is employed in the work on artificial intelligence. The expression was introduced by Gerardo Beni and Jing Wang in 1989. The algorithms have characteristics are of capabilities of self organizing system, adaptation to the changing conditions, self healing and local decision making. Using SI we can achieve more through put, data transmission and average efficiency. Territorial predators such as tigers, bears and dogs can be defined as predators that consistently defend a specific area against animals from other species. The territory is based on certain factors such as food resources. Most territory predators use scent marking to indicate the boundaries of their territories. Which are also playing a role in territorial maintains and as information sites for other members of the population. The rest of the paper is organized as follows, in section 2 related work, in section 3 describes the placement of sensor node using TPSMA, in section 4 problem statement, in section 5 TPSM algorithm, in section 6 LEACH protocol, in section 7 simulation and results and finally in section 8 describes conclusion and future work .

\section{RELATED WORK}

In the following words, we will study overview of placement of sensor node placement different techniques. Romoozi et al [1] stated that there is trade of between energy consumption of sensor nodes and network coverage. When placement of sensor nodes are closer, decrease in energy consumption but network coverage will smaller. This scenario helps full in deployment of sensor node. The energy consumption in wireless sensor network prevents the problem of the network exhausting of batteries, so partitioning the entire network into small parts. Kirnakumar et al [2] has designed the energy aware node placement algorithm for wireless sensor network 
communication technology that allows the random participation in the network. It helps in uncovered area, where fewer nodes located at far distances. The drawback is additional energy spent by the nodes located at dense location, By this calculating network life time. They also told that sensor node can be placed by placing nodes in rectangle or hexagonal. Yunxia chen et al [3] discovered the life time per unit cost defined as network life time can be measured by number of sensor node deployed in WSN. Placement sensor node near to the gateway node for reducing energy consumption for reporting. In this path loss exponent increases, the distance between adjacent sensors approaches uniform. Luis Daniel Samper Escalante et al [4] greedy routing algorithm for WSN presented that energy consumption takes part important in WSN by taking less hop numbers leads better performance. By using the swarm intelligence technique of ant colony optimization sending update to neighbors. When a critical event occurs does not negatively impact in the network. Selection of paths depends on few numbers of hops, less pheromone concentration to avoid over using of route. Pheromone can measure number of times traffic has passed through node. By this it gives the energy algorithm so have another option to route the packets. Ammar hawbani et al [5] has proposed the wireless network system for limited communication range of WSN. This addresses the problem of data routing based on sensor grouping. It provides deep insight about how to divide sensor of a network into separate independent groups and how to organize into independent groups. Xia Li et al [6] introduced the hierarchical routing protocols of particle swarm clustering algorithm and inter clustering algorithm for WSN. In this protocol is used is AECRP, this protocol function as increase the life time of network and maximizes the coverage area. Nojeong heo et al[7] a distributed self spreading algorithm for placement of sensor node by self in mobile wireless sensor node. The random distribution of nodes over the region of interest, because of deployment cost and time. It would not gives uniform distribution, use full for longer life time. Husan Zainol abidin et al [8] proposed the problem of sensor node placement solved by using multi objective optimization approach for sensor node placement. It requires because of the optimal decision due to the presence of the tradeoff between the energy consumption and coverage area. Wendi Rabiner Heinzelman et al [9] developed the energy efficient communication protocol for wireless micro sensor network. Low energy adaptive clustering hierarchy LEACH uses localized coordination to enable the robustness for dynamic network. This protocol allows evenly distribution of energy though out the sensor, so doubling use full systems lifetime of networks.

\section{CONCEPT OF PLACEMENT OF SENSOR} NODES USING TPSMA

In WSN, network performance is measured in terms of energy efficient. Now growing application of wireless network sensor node placement technique become major concern. So bio inspired mechanism is known as TPSMA for sensor mode placement. Predators are dogs, bears, tiger in the territory is defined for specific area based on certain factor such as food resources. The territory predator uses the scent marking behavior at the same areas of boundaries. It helps in territorial maintains. Scent matching indicates to differentiate from one animal to other of predator from intruders by identifying their scent. TPSM algorithm has the behavior adapted in designing the placement of sensor node placement technique. The territorial area indicate placement of sensor nodes. Using TPSMA algorithm we can achieve maximum connectivity, min energy consumption, max coverage, minimum uniformity. The marks indicated by animals in territorial area by rubbing parts of bodies such as chin and foot, urination, defecation. Using glands like vegetation flattering, scratching [12] to sensor node placement is recognized by marked territories, that identified by scent matching behavior.

\section{PROBLEM STATEMENT}

As we are dealing with sensor node placement in WSN applications, it consists of memory, transceiver, processor and battery. The low power energy sensor nodes capacity enables for limited coverage and communication range. Once sensor node is deployed, once again we cannot change or replace batteries of sensor node in critical application such as battle field, fire forest etc. so in order to use sensor node placement effectively by keeping in mind that energy utilization of sensor node is to be optimized. So design of WSN using sensor node placement technique is TPSMA with LEACH protocol. And most important thing is data communication takes more energy than processing. The measure of network life time is important, while network functioning in WSN application still now many algorithms, methodologies have been developed to effectively utilize the energy of nodes in sensor node. Now we are developing new algorithm called TPSMA with LEACH protocol.

\subsection{Existing Method}

The monitoring area of sensor node placement is divided into number of small square areas called monitored locations of sensor node. These monitored locations are called as potential locations of sensor node, each small square area are equipped with one sensor[12]. The area is obstacle free, each sensor has its own specific energy and energy consumed by each node effects on network life time. Energy consumption carrier out as follows first one for maintain sensor node in active state, second one for number of nodes receives data for transmitting to sink node. And third one is for transmission of energy on the path in which energy draws from sensor to sink node.

In this utilization of effective planning mechanism in arranging limited number of sensor nodes like in applications in precision agriculture, landslide monitoring, and forest fire detection can be designed with fixed number of sensor node 
within monitored area. When seriously considering cost effective placement technique, it is necessary to determine locations of sensor node and location of node strongly effect the energy consumption, sensing coverage and life time of network. So TPSMA is best suited for placing sensor in monitored location based on predator marking its territories with their scent or odors. Chemical or olfactory communication leaves message for their other animals to communicate, relatively long lasting and later identified by inconspecifics. In our exiting method placing node is static and they would not change their position according to requirement. And in our existing method have concentrated on coverage area than energy efficient network.

\subsection{Proposed Method}

In our proposed method, we are designing the energy efficient WSN for long life time. With TPSM algorithm LEACH protocol is used for equally distribution of energy in all nodes. And placements of sensor node are become dynamic as per the requirement. A performance study has been carried out by comparing the performance of the proposed technique with the minimax and lexicographic sensor node placement. Uniformity is a performance metric is used for estimate a WSN lifetime. Simulation results shown larger coverage and provide long lifetime as compared to the two schemes.

\section{TERRITORIAL PREDATOR SCENT MARKING}

\section{ALGORITHM}

TPSM algorithm is mainly focus on animal marking area by their odors or scent, urination for communication with their animals for certain factors such as food resources. The territory is selected based on food resources. Scent marking indicates boundaries of their territories [10]. Animal odors are used for communication between conspecifics according to four different functions such as scent matching, reproductive signaling and resource protection [12]. For eg Male tiger marks tress by spraying of urine and anal glands secretions. Dogs mark by their scent by urination defecation.

\subsection{Marking Phase}

Predator checks for all food resources on all ' $\mathrm{L}$ ' locations and marks the location with the highest food resources level Lmarked.

$$
\begin{gathered}
\forall=\{1,2, \ldots,\}:(\mid \in[0, \infty)) \\
\mathrm{Rx}=\mathrm{F}(\mathrm{x})
\end{gathered}
$$

Where $\mathrm{Rx}=$ food resources level at location $\mathrm{x}$.

$\mathrm{F}(\mathrm{x})=$ Objective Function value.

Lmarked $=$ marked Location.

Step1) Start by finding location for food resources.
Step2) Mark the food resources level at location $x=R x$.

Step3) If other any place have food resources, then find mark is present. If not sort all Rx.

Step4) For monitoring location first separate all Location into maximum sensor node area and Minimum sensor node in that area.

$$
\operatorname{Max}(\mathrm{Rx})=\text { Lmarked } .
$$
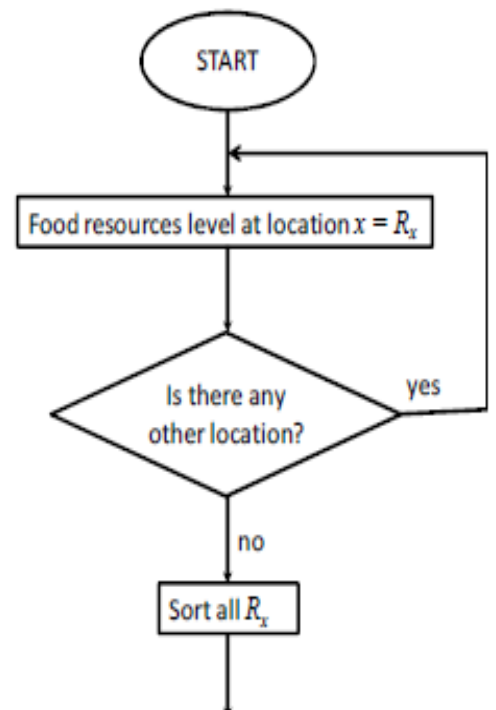

Mark location with highest food resource, $\max \left(R_{x}\right)=L_{\text {maxted }}$

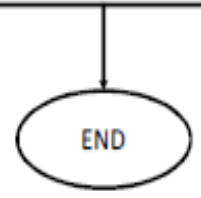

Fig - 1 Marked Phase

\subsection{Matching Phase:}

Predator locates the marked location Lmarked . Assume that the predator locates Lmarked based on linear search behavior as shown in the algorithm [18].

Procedure linear search(Lmarked : integer, R1, R2, ... RL: distinct integers)

$$
\begin{aligned}
& \mathrm{i}:=1 \\
& \text { while }(i \leq L \wedge \text { Lmarked } \neq R i) \\
& \mathrm{i}:=\mathrm{i}+1 \\
& \text { if } i \leq L \\
& \text { then location }:=i \\
& \text { else location }:=0 \\
& \text { return location }
\end{aligned}
$$

Step 1) Start with maximum sensor node area marked location.

Step 2) If location matched, then it identifies its location for communication. 
Step 3) If it is not of maximum marked location, then find marking for next maximum marked location of area.

Step 4) If totally no location of mark found for placing sensor node; end by finding sensor node placement.

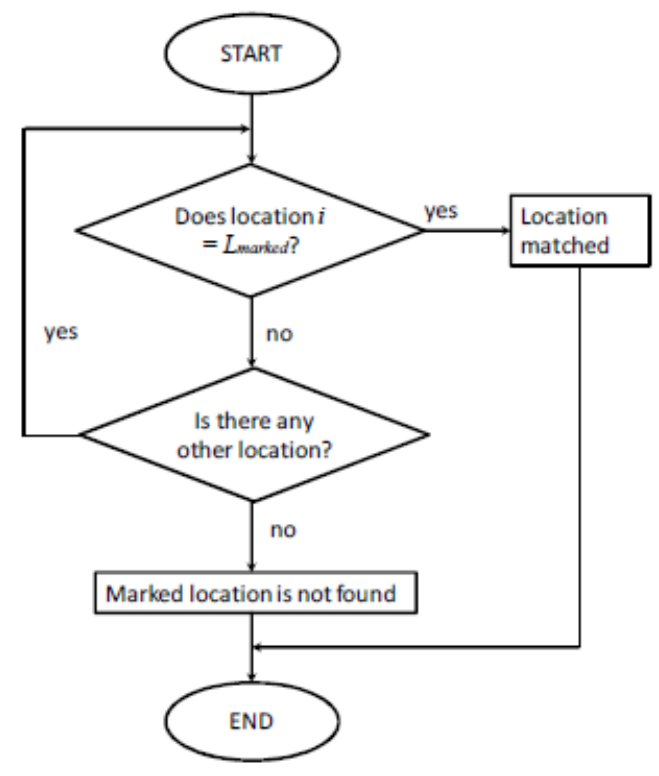

Fig -2 Matching phase

\section{LEACH PROTOCOL}

LEACH is used for load balancing, better energy utilization, ease of deployment and longer network life time[9].LEACH is a TDMA based MAC protocol, which is combined with clustering and simple routing protocol in WSNs. The main goal of LEACH is lower the energy consumption required to create and maintain clusters to increase the life time of WSN. it is a hierarchical protocol in which almost all nodes transmit to cluster heads. Functions of cluster heads are combining and compress the data and forward to the sink node. It is a randomized rotation of cluster heads among the sensor. All non cluster heads transmit data to cluster head. Cluster head receives the data and performs signal processing function on the data and transmit data to the base station. TDMA schedules first one prevents collision among data message. Second one in energy conservation in non cluster head nodes. The cluster head creates schedule for each node in its cluster heads only communicate with the cluster head in a TDMA. According to the schedule created by the cluster head. So using minimum energy needed to reach cluster head.

\section{SIMULATIONS AND RESULT}

The numerical simulation is done by using MATLAB for showing performance of proposed method. Simulation result are compared with results produced by minimax[20] and lexicographic minimax (lexmin)[21]

\section{Coverage Ratio:}

Coverage ratio of WSN for different number of sensor nodes. Coverage ratio of TPSMA with LEACH protocol is maximum than the previous normal TPSMA, lexmin and minimax.This means that maximum coverage ratio with minimum number sensor nodes and energy consumption of each sensor node are less. Lexmin and minimax are focus on the distance of sensor nodes with monitored location. Distance would not give maximum coverage area. Finally it indicates that with given energy in sensor nodes maximum coverage, so we conclude that TPSMA with LAEACH protocol is energy efficient sensor node placement technique.

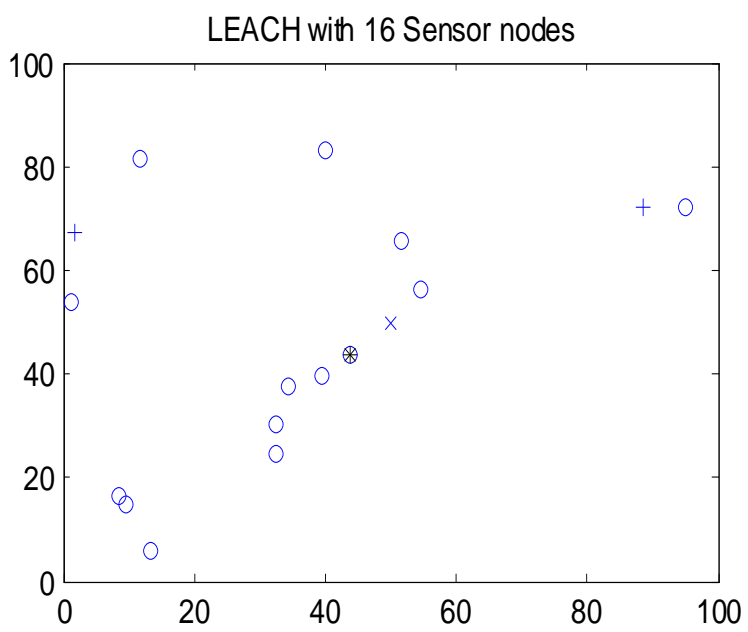

Fig -3 Placements of 16 sensor nodes

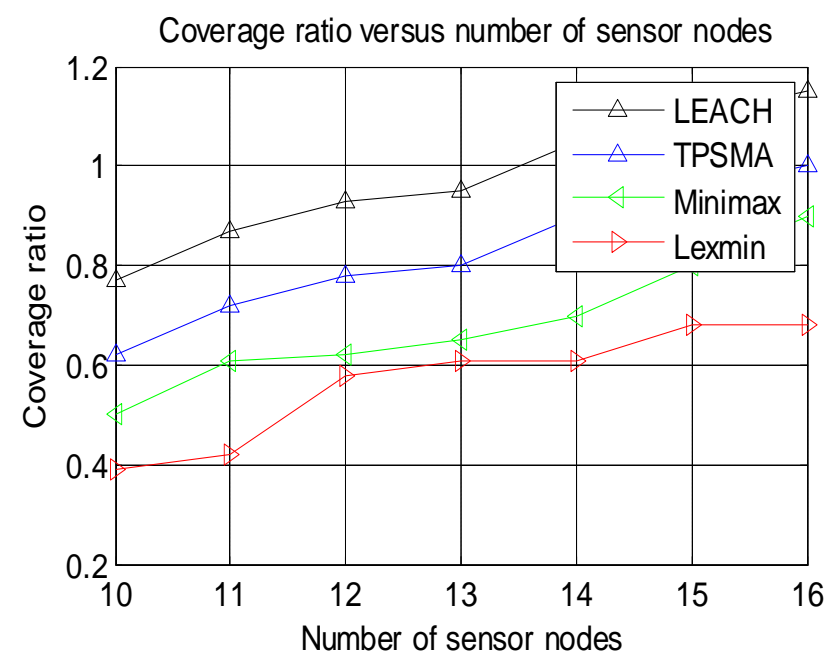

Fig - 4 Coverage ratios versus number of sensor nodes

\section{Sensor Node Position:}

Figure- 5 shows the 10,13 and 16 sensor node positions respectively. The red color indicates the access points of 
monitored locations, while black colors in the graph indicate the sensor nodes. The blue color circles indicate coverage area of sensor nodes.

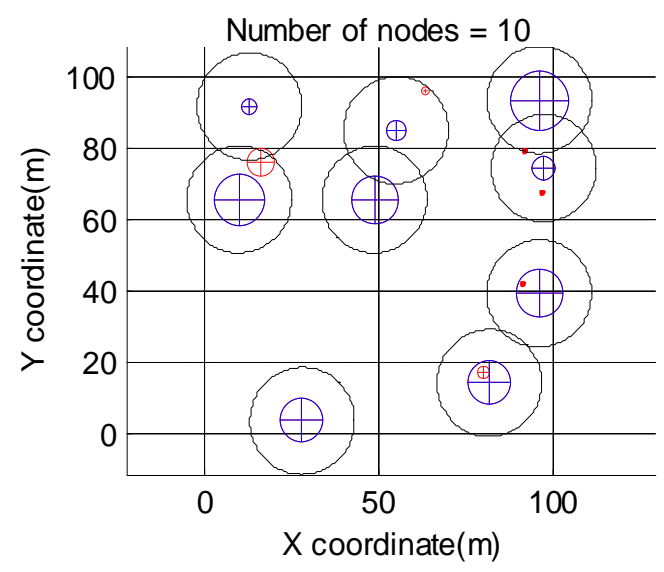

(a) 10 Sensor Nodes

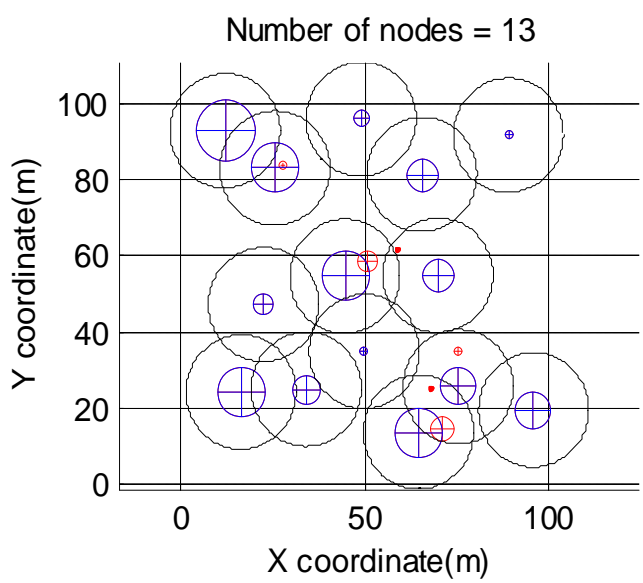

(b) 13 Sensor Nodes

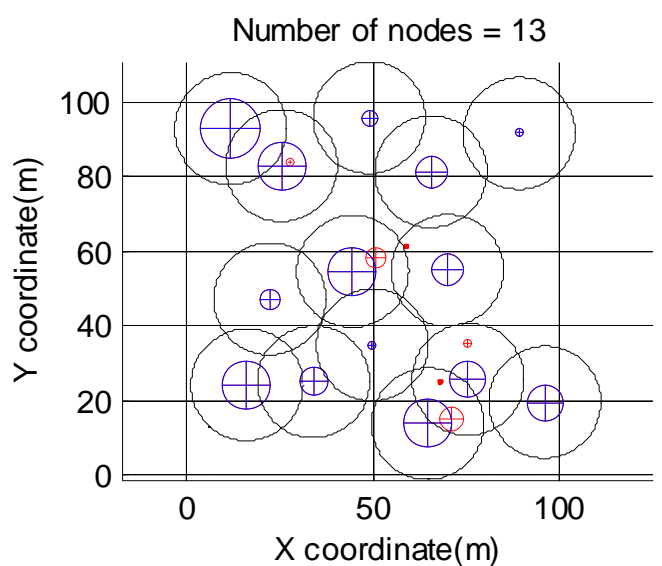

(c) 16 Sensor Nodes

Fig - 5 Sensor nodes positions

\section{Network Uniformity:}

Uniformity is used for measuring the system life time, where WSN sensor nodes defined as shortest life time of all sensor nodes. This is because a uniformly distributed sensor node spends energy more evenly through all nodes in the network .WSN with smaller uniformity shows more evenly distributed in the monitoring area. The mean distances for all nodes to their neighboring nodes in a uniformly distributed network are equal. And hence expected energy consumption per communication is lower, longer network life time.

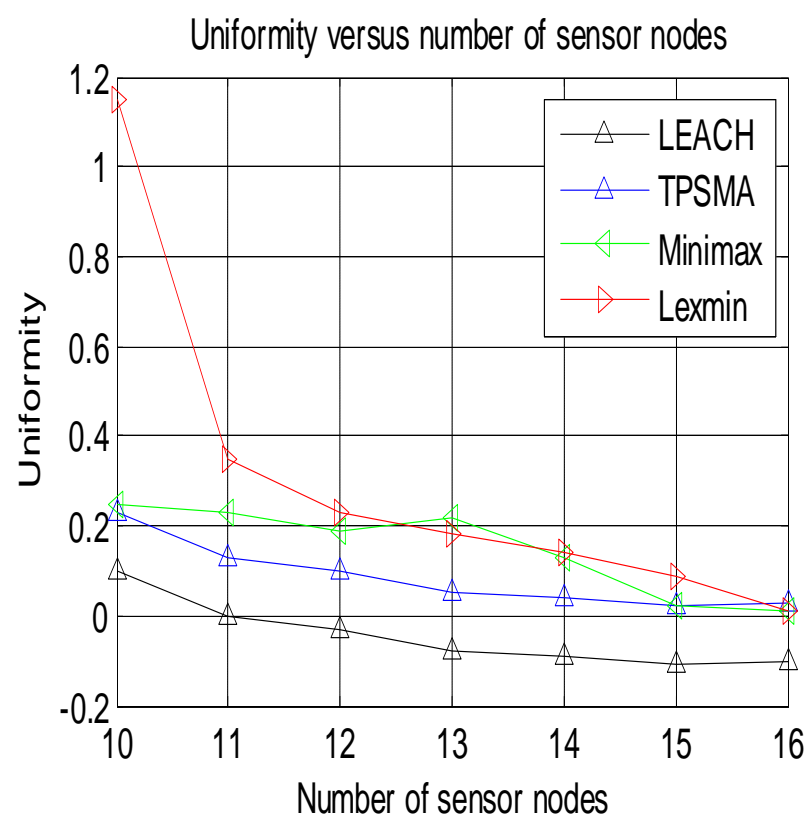

Fig - 6 Uniformity versus number of sensor nodes

\section{CONCLUSIONS AND FUTURE WORK}

In WSN sensor node placement based in TPSMA with energy balancing LEACH protocol has been proposed in order to increase the maximum coverage area, optimum less number of sensor nodes, energy efficient maximum network life time. The performance is compared with minmax and lexmin schemes. This network model consists of several equal widths monitored locations. Design of network is self organized functionality to dynamically adapt to its environment. By using Swarm intelligent TPSMA with LEACH protocol scheme outperformance the node scheduling scheme, proper protocol and efficiently utilizing energy save the power.

\section{REFERENCES}

[1]. M.Romoozi,M.Vahidipour and Maghsoodi, "Genetic Algorithm for Energy Efficient and Coverage-Preserved Positioning in Wireless Sensor Networks, " in proceedings of the international conference on intelligence computing and cognitive informatics(ICICCI'10).pp. 22-25, Kuala Lumper ,Malaysia june 2010. 
[2]. Kirankumar .Y and Jayashree D.Mallapur Basaveswar Engineering college Vidyanagar, Bagalkot. Advances in electronic and electric engineering ISSN 2231 -1297, volume 4Number 6(2014), ppp541-548 @ Research India Publication. [3]. "Sensor node placement for maximizing lifetime per unit cost in wireless sensor network,"Yunxia chan, chan-Nee chuah, and Qing zhao Department of electrical and computer Engineering university of California, Davis, CA .

[4]. "Swarm intelligence based energy saving greedy routing algorithm for wireless sensor networks" Escalante, L.D.S .ElectronicsCommunicationsandComputing(CONIELECOMP ),2013International Conference on digital Object Identifier: 10.1109/CONIELECOMP.2013.6525754.

[5]. "Wireless sensor network routing based on sensor grouping,"Ammar Hawbani, xingfu Wang,Yan Xinong , Saleem Karmoshi, University of Science and Technology of china,SchoolofcomputerscienceandTechnology,Hefei,china.E mail:ammar12@mail.ustc.edu.cn.2014,6,817(http://www.scirp .org/journal/wsn).

[6]. "An energy- efficient routing protocol based on PS clustering algoritm and inter-cluster routing algorithm for WSN," XiaLi ,Wang Gang ,Liu Zongori,Zhangyan yanuyan college of information Science and Engineering North EasternUniversity,Shanyang110819.Email:xiali@ise.neu.edu. cn; hurryican@163.com .

[7]. Nojeong Heo and Pramod K. Varshney."A Distributed SelfSpreadingAlgorithm,"Energy- Efficient Communication Protocol for Wireless Micro sensor Networks.

[8]. "Multi-objective Optimization (MOO) approach for sensor node placement in WSN ,"Abidin, H.Z.; Fac.of Electr.Engg,Univ.Teknol. MARA, Shah Alam, Malaysia; Din,N.M.; Jalil, Y.E. Signal Processing and Communication Systems (ICSPCS), 2013 7th International Conference. INSPEC Accession Number: 14061222.

[9]. "Wendi Rabiner Heinzelman, Anather chndrakasan, and haribalakrishnaanMassahusettsInstituteofTechnologyCambrid g,MA02139\{Wendi,anantha,hari\}@mit.edu .S. C. Huang, et al, (2011), proposes self-deploying approaches for sensor networks.

[10]. Begg C M, Begg K S, Du Toit J T and Mills M G L 2003 Scent-marking behaviour of the honeybadger, Mellivoracapensis (Mustelidae),inthesouthern Kalahari Animal Behaviour 66 917-929.

[11]. Energy efficient sensor nodes placement using TerritorialPredatorScent Marking Algorithm (TPSMA)H Z Abidin1 and N M Din2, Faculty of Electrical Engineering, Universiti Teknologi MARA, 40450 Shah Alam,Selangor, MalaysiaCollegeofEngineering,UniversitiTenagaNasional,jala nIKRAMUNITEN,43000.Kajang,Selangor,MalaysiaEmail:hu snaza@salam.uitm.edu.my .

[12]. K. A. Descovich, A. T. Lisle, S. Johnston, V. Nicolson,

C. J. C. Phillips, "Differential responses of captive southern hairy-nosed wombats (Lasiorhinus latifrons) to the presence of faeces from different species and male and female conspecifics," Applied Animal Behaviour Science,Vol. 138, pp. 110-117, 2012. 